\title{
Glutamine synthetase mediates sorafenib sensitivity in $\beta$-catenin-active hepatocellular carcinoma cells
}

\author{
Bo Hwa Sohn ${ }^{1,2}$, In Young Park ${ }^{2}$, Ji-Hyun Shin ${ }^{1}$, Sun Young Yim ${ }^{1,3}$ and Ju-Seog Lee ${ }^{1}$
}

The gene encoding $\beta$-catenin is frequently mutated in hepatocellular carcinoma cells. While the oncogenicity of $\beta$-catenin has been extensively studied, $\beta$-catenin's role in hepatocellular carcinoma tumor metabolism is currently less well understood. In this study, we found that $\beta$-catenin regulates the expression of glutamine synthetase and triggers a series of metabolic changes leading to induction of autophagy in hepatocellular carcinoma cells. $\beta$-Catenin-active Hep3B and HepG2 cells exhibited higher basal levels of autophagic activity than did $\beta$-catenin wild-type cells. We also found that autophagy in $\beta$-catenin-active cells was mediated by glutamine synthetase, as silencing of glutamine synthetase significantly reduced autophagic activity. We also showed that $\beta$-catenin-active hepatocellular carcinoma cells were more sensitive to sorafenib than were $\beta$-catenin wild-type cells. Our results demonstrated that glutamine synthetase-mediated autophagy explains the high sensitivity of $\beta$-catenin-active hepatocellular carcinoma cells to sorafenib. Our results highlight the importance of glutamine metabolism in the regulation of autophagy in hepatocellular carcinoma cells. More importantly, our study unravels the molecular mechanisms leading to sorafenib sensitivity in hepatocellular carcinoma.

Experimental \& Molecular Medicine (2018) 50, e421; doi:10.1038/emm.2017.174; published online 5 January 2018

\section{INTRODUCTION}

Hepatocellular carcinoma (HCC) is one of the deadliest cancers in the world. HCC tumors are often advanced at diagnosis, and treatment options are limited. Therefore, overall mortality rates for HCC patients are higher than those for patients with many other cancers. ${ }^{1-3}$ At present, surgical resection is the preferred therapy for HCC. However, only about $15 \%$ of HCC patients are eligible for surgical resection, and up to $70 \%$ of patients who undergo resection experience disease recurrence within 5 years of surgery. ${ }^{4}$ Therefore, development of more individualized and effective systemic therapies is necessary for HCC patients who are not eligible for surgery and those with recurrence after surgery. ${ }^{5}$ Sorafenib, currently the only approved drug for first-line systemic treatment of advanced HCC, is an orally active multikinase inhibitor. While randomized phase III trials demonstrated that sorafenib has clinically significant anticancer activity, ${ }^{6,7}$ its survival benefits were only modest, suggesting that not all patients benefit from sorafenib treatment.

The liver's physiological homeostasis is maintained through high levels of metabolic activity in hepatocytes, which is regulated through structural and functional units of the liver called hepatic lobules. The hepatocytes within a lobule express different transcriptional programs depending on which metabolic zone they are located in. ${ }^{8}$ Hepatocytes in the periportal metabolic zone express genes involved in gluconeogenesis and fatty acid metabolism, whereas hepatocytes in the perivenous zone express genes involved in detoxification. $\beta$-catenin is a master regulator of metabolic zonation in the liver, and its activity is limited in the perivenous zone, where it maintains the perivenous-specific transcriptome program. ${ }^{9}$ Glutamine synthetase (GS) is a metabolic enzyme that produces glutamine from glutamate and ammonia and is involved in detoxification of ammonia in the liver. Because GS is part of the perivenous transcriptome, its expression is restricted to hepatocytes in the perivenous zone and is tightly regulated by $\beta$-catenin. ${ }^{9-11}$ The gene encoding $\beta$-catenin, CTNNB1, is a frequently activated oncogene in HCC; its aberrant activation is largely attributable to somatic mutations, which occur in 20-30\% of HCC patients. ${ }^{10-13}$ In the current study, we discovered unexpected roles of $\beta$-catenin and its downstream metabolic target gene that may be associated with treatment response in HCC patients.

${ }^{1}$ Department of Systems Biology, The University of Texas MD Anderson Cancer Center, Houston, TX, USA; ${ }^{2}$ Department of Cellular and Molecular Biology and Medicine, Center for Precision Environmental Health, Baylor College of Medicine, Houston, TX, USA and ${ }^{3}$ Division of Gastroenterology and Hepatology, Department of Internal Medicine, Korea University School of Medicine, Seoul, Korea

Correspondence: Dr J-S Lee, Department of Systems Biology, The University of Texas MD Anderson Cancer Center, Division of Cancer Medicine, Unit 950, 1515 Holcombe Blvd., Houston, TX 77054, USA.

E-mail: jlee@mdanderson.org

Received 4 April 2017; accepted 11 May 2017 


\section{MATERIALS AND METHODS}

\section{Cell lines and cell culture}

SK-Hep1, HepG2, Hep3B and Huh7 human HCC cells were obtained from the Health Science Research Resources Bank (Osaka, Japan), and FOCUS HCC cells were kindly provided by Dr Snorri S. Thorgeirsson at the National Cancer Institute (Bethesda, MD, USA). The cells were maintained in Dulbecco's modified Eagle's medium with high glucose and $4 \mathrm{mM}$ glutamine (Invitrogen, Carlsbad, CA, USA) supplemented with heat-inactivated $10 \%$ fetal bovine serum and penicillin/streptomycin (Invitrogen).

\section{Lentiviral GS overexpression vector and short hairpin RNAs} A GS-overexpressing lentiviral vector was constructed by cloning a full-length coding DNA fragment into the NheI/NotI sites of a pCDHEF1-T2A-Puro vector (System Biosciences, Mountain View, CA, USA). Lentiviral-based short hairpin RNAs (shRNAs) targeting human GS (NM_002065.3-802s1c1, NM_002065.3-1450s1c1 and NM_002065.4-1890s21c1) and control shRNA targeting enhanced green fluorescent protein (GFP) (cat. no. SHC005V) were used for knockdown experiments.

\section{Lentiviral transduction}

To produce lentiviral particles, the GS overexpression vector or shRNA expression vectors were co-transfected with the lentiviral packaging plasmids pLP1, pLP2 and pLP/VSVG (Invitrogen) into 293FT lentivirus packaging cells. Lipofectamine 2000 (Invitrogen) was used as the transfection reagent. At $48-72 \mathrm{~h}$ after transfection, the virus-containing cell culture medium was harvested and frozen in aliquots. A moderate multiplicity of infection was used for transduction of cells to minimize negative effects on cell proliferation. All experiments were performed 2-4 days after infection
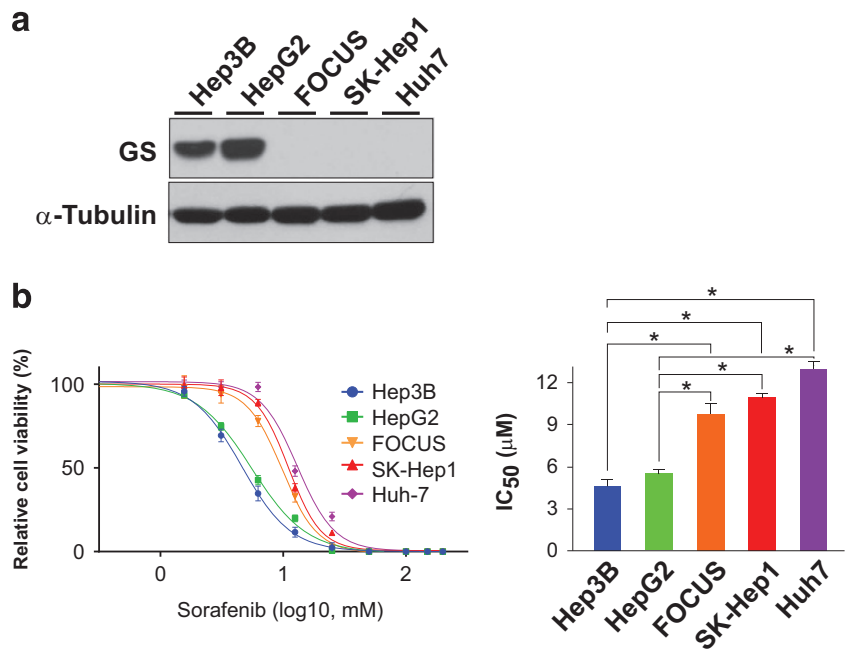

Figure 1 Sensitivity to sorafenib is associated with glutamine synthetase (GS) expression levels in hepatocellular carcinoma (HCC) cells. (a) Representative western blots showing expression of GS in five HCC cell lines. (b) Results of cell viability assays performed $48 \mathrm{~h}$ after treatment with the indicated doses of sorafenib. $I_{50}$ values were compared using a Student $t$ test. Data shown are means \pm s.e's. of the mean from three independent experiments. ${ }^{*} P<0.01$.

\section{Antibodies}

Antibodies against phospho-p70 S6 kinase (Thr389), p70 S6 kinase, phospho-S6 (Ser235/236), S6, LC3B and $\alpha$-tubulin were purchased from Cell Signaling Technology (Danvers, MA, USA; cat. nos. 9205, 9202, 4858, 2217, 2775 and 3873). Antibodies against GS were obtained from EMD Millipore (Danvers, MA, USA; cat. no. MAB302). Antibodies against $\beta$-actin were purchased from SigmaAldrich (St. Louis, MO, USA; cat. no. A5441). Horseradish peroxidase-conjugated anti-mouse immunoglobulin $\mathrm{G}$ and horseradish peroxidase-conjugated anti-rabbit immunoglobulin $G$ were purchased from Santa Cruz Biotechnology (Santa Cruz, CA, USA; cat. nos. sc-2748 and sc-2749).

MTT cell viability assay. To assess cell viability. A 3-(4,5dimethylthiazol-2-yl)-2,5-diphenyltetrazolium bromide (MTT) assay (Sigma-Aldrich) was performed as described previously ${ }^{5,6}$ using a VMax kinetic microplate reader (Molecular Devices, Sunnyvale, CA, USA) at $570 \mathrm{~nm}$. Cells were fixed in $10 \%$ formaldehyde and stained with $10 \%$ crystal violet (Sigma-Aldrich) for $10 \mathrm{~min}$ at ambient temperature.

\section{Flow cytometry}

Samples were collected at $48 \mathrm{~h}$ after sorafenib treatment and fixed in $70 \%$ ethanol overnight. To measure the proportion of apoptotic cells, we used an Annexin V apoptosis detection kit (Santa Cruz Biotechnology) according to the manufacturer's protocol. Cells were stained with Annexin V-fluorescein isothiocyanate and DAPI after sorafenib treatment. Cells were evaluated for apoptosis via flow cytometry using an LSR II flow cytometer (BD Biosciences, San Jose, CA, USA).

\section{Autophagic puncta analysis}

SK-Hep1 cells were stably transfected with pEGFP-LC3 and pDsRed2C1 or pDsRed2-GS constructs and plated on coverslips. The plated cells were exposed to sorafenib for $24 \mathrm{~h}$ and then fixed with $4 \%$ paraformaldehyde in phosphate-buffered saline. Autophagic puncta were counted using an ECLIPSE TE2000-E fluorescence microscope (Nikon, Melville, NY, USA).

\section{RESULTS}

\section{$\boldsymbol{\beta}$-catenin-active HCC cells are sensitive to sorafenib}

Because expression of GS is tightly regulated by $\beta$-catenin in perivenous hepatocytes and reflects $\beta$-catenin activity, ${ }^{9-11}$ we determined the activation status of $\beta$-catenin by assessing GS expression in five HCC cell lines. HepG2 cells were used as positive controls for assessments of $\beta$-catenin activation because $\beta$-catenin in HepG 2 cells is known to be constitutively activated by deletion mutations in negative regulatory sites. ${ }^{13}$ As expected, GS was highly expressed in HepG2 cells (Figure 1a). GS was also highly expressed in Hep3B cells, suggesting that $\beta$-catenin is highly active in this cell line. This observation is in good agreement with a previous study demonstrating that $\beta$-catenin is highly active in Hep3B and that the tumorigenicity of xenografted Hep3B cells depends on $\beta$-catenin expression. ${ }^{14}$ In contrast, expression of GS was absent in FOCUS, SK-Hep1 and Huh7 cells, indicating that $\beta$-catenin is not active in these cell lines.

HCC with activated $\beta$-catenin has a distinct clinical course; studies showed that HCC patients with activated $\beta$-catenin 
have better prognoses than do those with wild-type $\beta$ catenin. ${ }^{15,16}$ Therefore, we determined whether $\beta$-cateninactive HCC cells and HCC cells with wild-type $\beta$-catenin responded differently to sorafenib. ${ }^{6,7}$ To measure the half maximal inhibitory concentration $\left(\mathrm{IC}_{50}\right)$ of sorafenib in each HCC cell line, we treated five HCC cell lines with various concentrations of sorafenib for $48 \mathrm{~h}$ and counted the viable cells. Interestingly, the two HCC cell lines with activated $\beta$ catenin, HepG2 and Hep3B, were more sensitive to sorafenib than were those with inactive $\beta$-catenin activity (Figure $1 b$ ). The $\mathrm{IC}_{50}$ values of sorafenib in $\beta$-catenin-active HepG2 and Hep3B cells were two to three times lower than in cell lines with inactive $\beta$-catenin $(P<0.001$ for all comparisons), suggesting a link between $\beta$-catenin activation and sensitivity to sorafenib.

\section{Sensitivity to sorafenib in $\beta$-catenin-active HCC cells is mediated by GS}

Because the most distinctive liver-specific function of $\beta$-catenin is control of glutamine metabolism through expression of GS, ${ }^{17}$ we next tested whether GS plays a role in sorafenib sensitivity in $\beta$-catenin-active HCC cells. First, we assessed sorafenib sensitivity after silencing GS expression with lentiviral shRNAs in GS-high HCC cell lines (HepG2 and Hep3B). When GS was depleted by transduction with two GS-specific shRNAs, HepG2 cells develop resistant to sorafenib (Figure $2 \mathrm{a}$ ). The $\mathrm{IC}_{50}$ value of sorafenib increased from $5.4 \mu \mathrm{M}$ in parental HepG2 cells to $9.1 \mu \mathrm{M}$ and $9.9 \mu \mathrm{m}$ (both $P<0.001$ ) in HepG2 cells with silenced GS expression $(n=3)$. Similarly, depletion of GS via transduction with two shRNAs made Hep3B cells resistant to
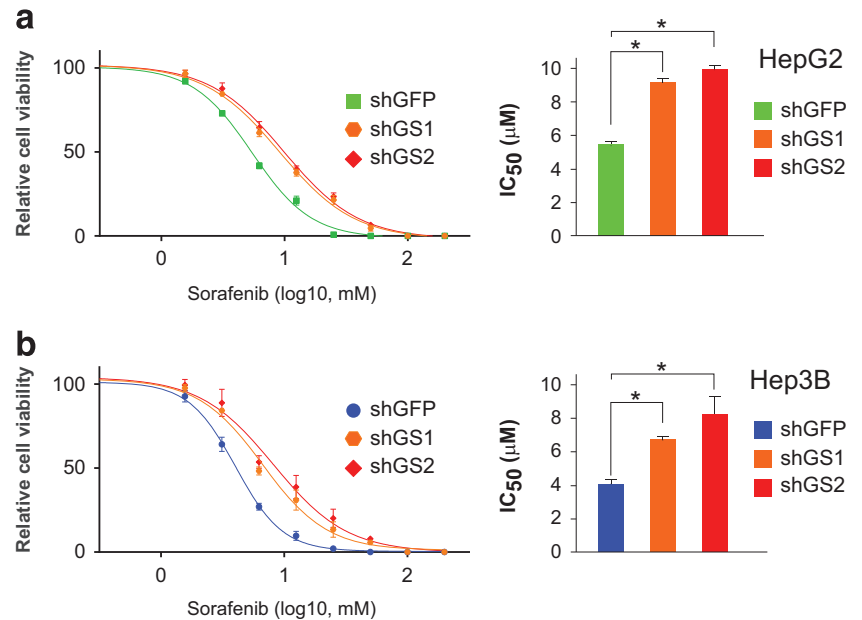

Figure 2 Depletion of glutamine synthetase (GS) expression decreased sorafenib sensitivity in hepatocellular carcinoma (HCC) cells. Results of cell viability assays performed $48 \mathrm{~h}$ after sorafenib treatment in HepG2 (a) and Hep3B (b) cells transduced with GSsilencing shRNAs (shGS1, shGS2) or a control shRNA against green fluorescent protein (shGFP). $I_{50}$ values were compared using a Student $t$ test. Data shown are means \pm s.e's. of the mean from three independent experiments. ${ }^{*} P<0.001$. sorafenib $(P<0.001$; Figure $2 \mathrm{~b})$, strongly suggesting that GS regulates the sensitivity of HCC cells to sorafenib.

We next overexpressed GS in GS-low SK-Hep1 cells by transducing them with a lentivirus harboring an exogenous GS gene. We examined the viability of the transduced cells after $48 \mathrm{~h}$ of sorafenib treatment. GS overexpression sensitized SK-Hep1 cells to sorafenib (Figure 3a). The $\mathrm{IC}_{50}$ value decreased from $11.9 \mu \mathrm{m}$ in parental SK-Hep1 cells to $4.7 \mu \mathrm{M}$ in GS-overexpressing cells $(n=3 ; P<0.001)$. GS overexpression also significantly sensitized FOCUS cells to sorafenib (Figure $3 \mathrm{~b} ; P<0.001$ ), confirming that the sensitivity of $\beta$-catenin-active HCC cells to sorafenib is mediated by GS.

Finally, we examined apoptosis rates in GS-overexpressing SK-Hep1 cells using flow cytometry. Sorafenib treatment induced apoptosis at a significantly higher rate in GSoverexpressing SK-Hep1 cells than in low-GS parental SKHep1 cells $(P<0.001$, Figure $3 c$ and $d)$. Because exogenous overexpression of GS had little effect on the apoptosis rate in untreated SK-Hep1 cells, it is unlikely that the observed increase in sensitivity to sorafenib could be attributed to GSmediated toxicity in HCC cells. Therefore, these results suggested that sensitivity to sorafenib strongly depends on GS expression levels in HCC cells.

\section{GS induces autophagy}

A previous study showed that increased intracellular production of glutamine was correlated with induction of autophagy, ${ }^{18}$ suggesting that $\beta$-catenin-active HCC cells should exhibit high levels of autophagy. Autophagic activity is also known to be high in the perivenous zone, where $\beta$-catenin is highly active. ${ }^{19}$ Thus, we next tested whether $\beta$-catenin induces autophagy in HCC cells.

We estimated basal autophagy levels by measuring the conversion of microtubule-associated light chain 3 (LC3-I) to its lipidated form (LC3-II), which is a key step in autophagosome formation and a biomarker of autophagy initiation. ${ }^{20}$ As expected, basal levels of autophagic activity as represented by the LC3-I/LC3-II ratio were substantially higher in $\beta$-catenin-active Hep3B cells than in the other cell lines (Figure 4a). The LC3-II level in Hep3B cells was several times higher than that in SK-Hep1 and FOCUS cells. Because GS is a major metabolic target of $\beta$-catenin in HCC cells, we next determined whether the high levels of GS expression in Hep3B cells accounted for their high basal level of autophagic activity. We assessed autophagic activity by measuring the LC3-II/LC3-I ratio in GS-silenced Hep3B cells and GS-overexpressing SK-Hep1 cells. Depletion of GS in Hep3B cells by transduction with two independent shRNAs reduced LC3-II formation (Figure 4b). Conversely, exogenous GS expression increased autophagic activity in SK-Hep1 cells (Figure 4c). To verify autophagy induction, we generated a transient construct that expressed GFP)LC3 and transfected it into SK-Hep1 cells. Fluorescence microscopy showed that GFP-LC3 was expressed diffusely in the cytoplasm of mock-transfected SK-Hep1 cells. However, 

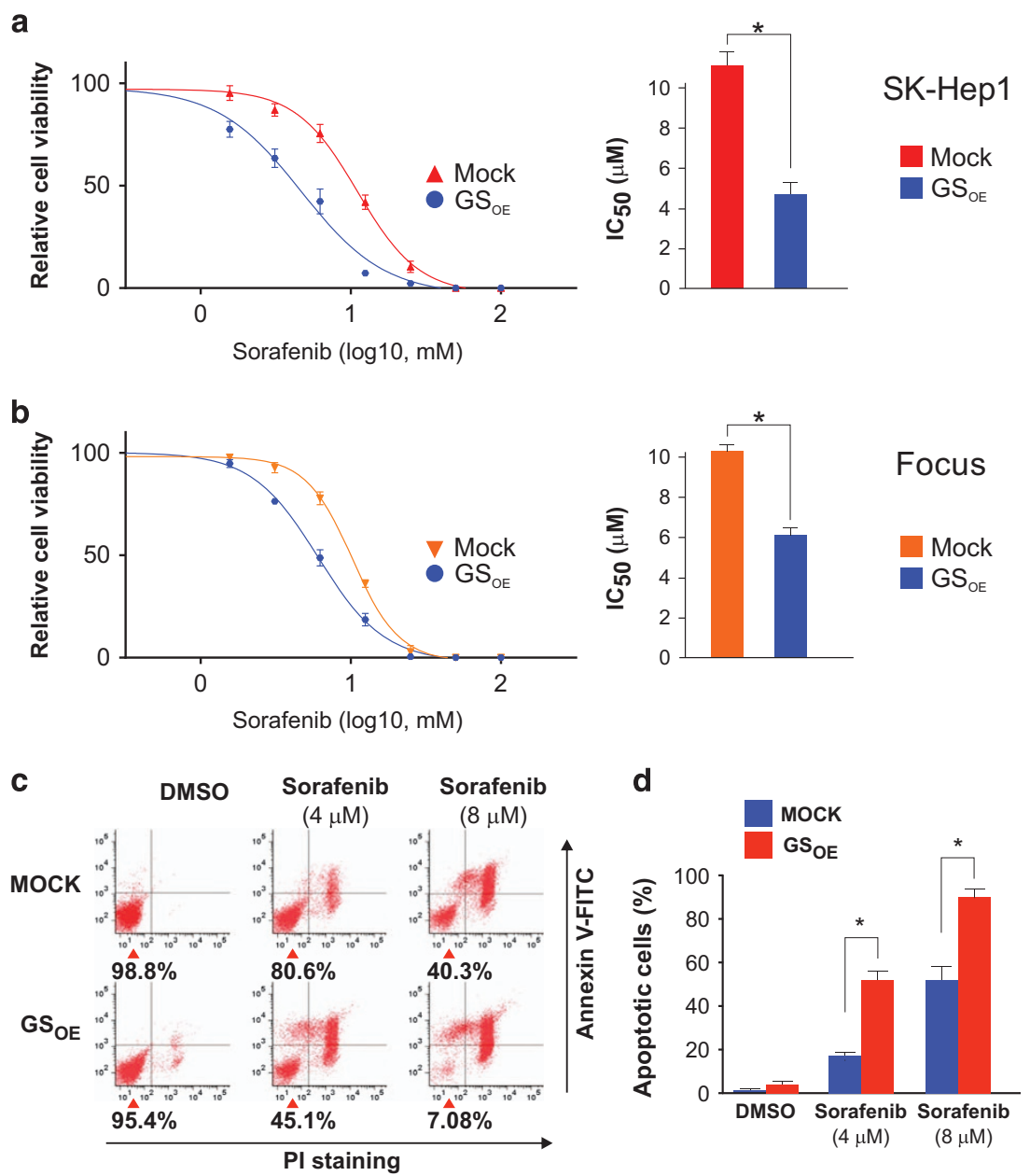

Figure 3 Glutamine synthetase (GS) overexpression significantly increased sorafenib sensitivity in hepatocellular carcinoma (HCC) cells. (a, b) Results of cell viability assays performed $48 \mathrm{~h}$ after sorafenib treatment in GS-overexpressing (GS OE) or mock-transfected (Mock) SkHep1 and FOCUS cells. IC 50 values were compared using a Student $t$ test. Data shown are means \pm s.e's. of the mean from three independent experiments. ${ }^{*} P<0.001$. (c) Results of flow cytometry apoptosis assays using Annexin V-FITC and DAPI staining in SK-Hep1 cells with $\left(\mathrm{GS}_{\mathrm{OE}}\right)$ or without $(\mathrm{MOCK})$ overexpression of GS after treatment with the indicated concentrations of sorafenib or dimethyl sulfoxide (DMSO) control. (d) Quantified flow cytometry data from three independent experiments. Data are presented as means \pm s.e's. of the mean. $P$ values were determined by Student $t$ test.

GFP-LC3 formed autophagic puncta in GS-expressing SKHep1 cells (Figure 4d), indicating that the increased formation of autophagosomes was mediated by GS. These results suggest that GS expression induces autophagy in HCC cells.

\section{Sorafenib sensitivity is mediated by autophagy}

The results of previous studies indicate that sorafenib can induce autophagy in HCC cells and that excessive autophagy can induce cell death. ${ }^{21,22}$ Thus, we hypothesized that the increase in sorafenib sensitivity observed in GS-expressing HCC cells is largely attributable to excessive autophagy that is jointly mediated by GS and sorafenib. Consistent with the results of previous reports, ${ }^{21,22}$ we found that sorafenib treatment increased autophagic activity in Hep3B cells and that GS depletion via knockdown using shRNAs substantially reduced sorafenib-induced autophagy (Figure 5a). These findings strongly suggest that the reduction in autophagic activity caused by GS inactivation explains resistance to sorafenib in HCC cells. To validate this conclusion, we overexpressed GS in SK-Hep1 cells and measured LC3-II using western blot analysis. Consistently, expression of the autophagy marker LC3-II was substantially increased by exogenous overexpression of GS (Figure 5b), and this increase in autophagic activity coincided with increased sorafenib sensitivity in SKHep1 cells (Figure 3a). These findings suggest that the high sensitivity of $\beta$-catenin-active HCC cells to sorafenib is mediated by their high basal level of GS-induced autophagy. In agreement with the results of the western blot analysis, analysis of autophagic puncta demonstrated that exogenous GS overexpression increased GFP-LC3 punctate formation in sorafenib-treated SK-Hep1 cells (Figure 5c). These results further validated our conclusion that the increased formation of autophagosomes was indeed mediated by GS. Taken 

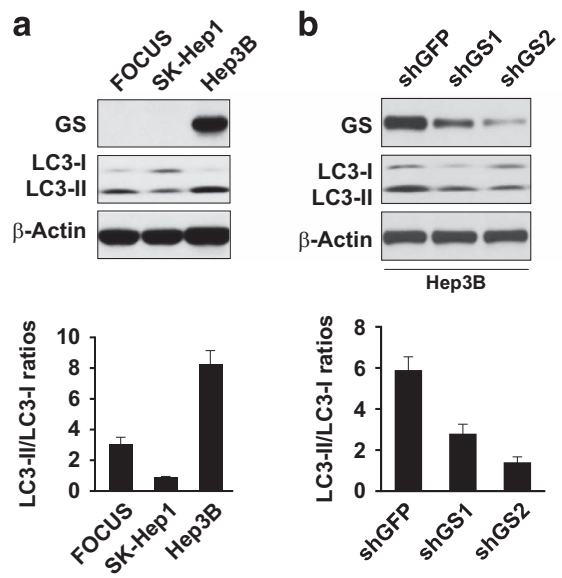
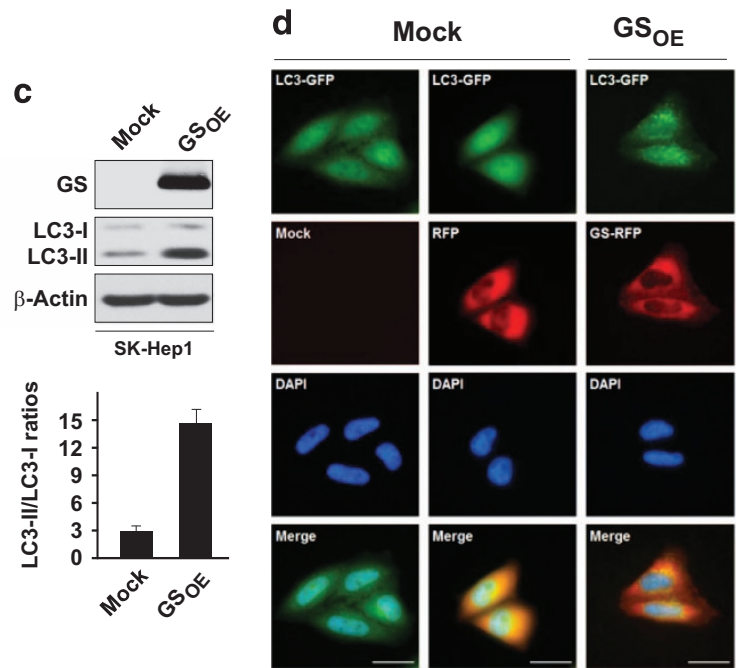

Figure 4 Glutamine synthetase (GS) activates autophagy in hepatocellular carcinoma (HCC) cells. (a) Western blots showing that high-GS Hep3B cells had higher LC3-II/LC3-I ratios than did low-GS FOCUS and SK-Hep1 cells. Expression of GS and autophagy markers LC3-I and LC3-II in HCC cells was assessed using western blotting. The LC3-II/LC3-I ratios and LC3-II levels were analyzed by quantitative densitometry ( $n=3$ independent experiments). (b) Western blots showing that GS knockdown decreased autophagic activity in Hep3B cells. Autophagy was measured by western blotting for LC3-I and LC3-II in Hep3B cells after GS expression was depleted by transduction with shRNAs shGS1, shGS2 or shGFP. The LC3-II/LC3-I ratios and LC3-II levels were analyzed as in a. (c) Western blots showing increased autophagic activity in GS-overexpressing (GSOE) SK-Hep1 cells. The LC3-II/LC3-I ratios were measured as in a. (d) Representative cell images showing autophagic puncta as speckles of LC3-GFP in GS-overexpressing SK-Hep1 cells. SK-Hep1 cells were stably transfected with pEGFP-LC3 (LC3-GFP), pEGFP-LC3 and pDsRed-C1 (RFP), or pEGFP-LC3 and pDrRed-GS (GFP-LC3) and analyzed using fluorescence microscopy. Scale bars, $20 \mu \mathrm{m}$.
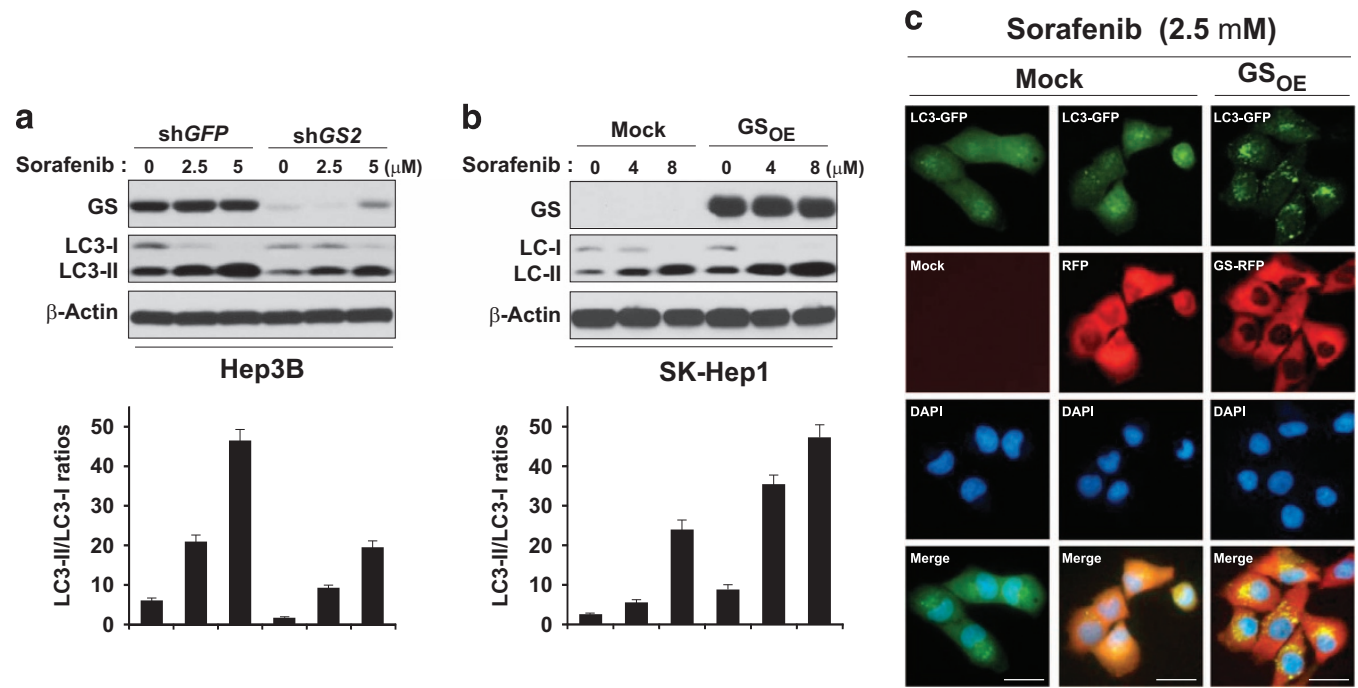

Figure 5 Glutamine synthetase (GS) expression augments autophagy induced by sorafenib treatment. (a) Expression of autophagy markers LC3-I and LC3-II induced by sorafenib treatment was assessed using western blotting. Autophagic activity was decreased by GS knockdown with shGS2 RNA in Hep3B cells. shGFP was used as a control. Cells were treated with sorafenib at the indicated concentrations for $48 \mathrm{~h}$. The LC3-II/LC3-I ratios and LC3-II levels were measured by quantitative densitometry ( $n=3$ independent experiments). (b) Western blots showing that autophagic activity induced by sorafenib treatment was increased by GS overexpression in SKHep1 cells. The LC3-II/LC3-I ratios were measured as in a after induction of GS overexpression. (c) Representative fluorescence microscopy images showing autophagic puncta as speckles of LC3-GFP after sorafenib treatment in GS-overexpressing SK-Hep1 cells. SKHep1 cells were stably transfected with pEGFP-LC3 (LC3-GFP), pEGFP-LC3 and pDsRed-C1 (RFP), or pEGFP-LC3 and pDrRed-GS (GFPLC3) and treated with $2.5 \mu \mathrm{m}$ sorafenib for $24 \mathrm{~h}$. Scale bars, $20 \mu \mathrm{m}$.

together, these results strongly support the notion that sorafenib sensitivity in $\beta$-catenin-active HCC cells is mediated by GS, which triggers a high basal level of autophagy.

\section{DISCUSSION}

In the current study, we identified a novel GS-mediated mechanism by which $\beta$-catenin induces autophagy. This 
finding is similar to recent findings that the oncogenes $M Y C$ and $R A S$ can stimulate autophagy. ${ }^{23,24,25}$ Because autophagy is activated to relieve cells in stress conditions, such as those under metabolic or environmental stress, autophagy generally promotes cell survival. In fact, autophagy induction by oncogenic RAS is necessary for cancer cell survival and oncogenic transformation. ${ }^{26}$ Our data strongly suggest that $\beta$ catenin activation relieves stress in HCC cells and promotes cell survival through activation of a liver-specific transcriptome involving GS.

Although sorafenib is the only approved systemic drug for the treatment of patients with HCC, its efficacy is limited, as evidenced by the only 2- to 3-month increase in survival duration observed in clinical studies. ${ }^{6,7}$ Only a small subset of patients experiences a response to treatment. Sorafenib is well known to exert its antitumor effects by inhibiting several kinases. It also induces autophagy as other chemotherapy agents. $^{21,22}$ While autophagy is best known as a pro-survival mechanism, recent studies have shown that prolonged autophagy impedes cell recovery and survival, triggering cell death. ${ }^{27,28}$ HCC cells with high levels of GS expression may be particularly sensitive to sorafenib because they already have high basal levels of autophagy. Our data clearly demonstrated that $\beta$-catenin-activated HCC cells were more sensitive to sorafenib. We further demonstrated that sensitivity to sorafenib was mediated by high GS expression, which is a direct downstream target of $\beta$-catenin in HCC cells.

Taken together, our data suggest that HCC with activated $\beta$ catenin is not only a genetically distinct subtype of HCC but also a clinically distinct subtype of this disease. Understanding how a tumor's genetic subtype may affect its sensitivity to standard treatment may be useful for guiding treatment decisions. For instance, patients with HCC with activated $\beta$ catenin mutations may be more likely to benefit from sorafenib treatment.

In summary, we uncovered a connection between a liverspecific transcriptome regulated by $\beta$-catenin and the sensitivity of HCC cells to sorafenib, the standard treatment for HCC. Our results highlight the importance of glutamine metabolism in the regulation of autophagy in HCC cells. When activated by oncogenic mutations that lead to hepatotumorigenesis, $\beta$ catenin also activates a liver-specific transcriptome program that produces GS overexpression in HCC cells. More importantly, high GS expression in $\beta$-catenin-active HCC increases autophagy by modulating metabolic flux, which contributes to increased sorafenib sensitivity. Collectively, our results suggest that $\beta$-catenin-regulated metabolic activity may dictate clinical outcomes for patients with HCC.

\section{CONFLICT OF INTEREST}

The authors declare no conflict of interest.

\section{ACKNOWLEDGEMENTS}

This study was supported in part by the National Institutes of Health through Cancer Center Support Grant P30 CA016672, the Duncan Cancer Prevention Research Seed Funding Program (2016 cycle) and
MD Anderson Sister Institute Network Fund (2012 and 2016 cycles). Authors also thank to Dr Amy Ninetto at Department of Scientific Publications for editing the manuscript.

\section{PUBLISHER'S NOTE}

Springer Nature remains neutral with regard to jurisdictional claims in published maps and institutional affiliations.

1 Minguez B, Tovar V, Chiang D, Villanueva A, Llovet JM. Pathogenesis of hepatocellular carcinoma and molecular therapies. Curr Opin Gastroenterol 2009; 25: 186-194.

2 El-Serag HB. Hepatocellular carcinoma. N Engl J Med 2011; 365 : 1118-1127.

3 El-Serag HB, Mason AC. Rising incidence of hepatocellular carcinoma in the United States. N Engl J Med 1999; 340: 745-750.

4 Bruix J, Sherman M, Practice Guidelines Committee AAftSoLD. Management of hepatocellular carcinoma. Hepatology 2005; 42: 1208-1236.

5 Cabibbo G, Craxi A. Hepatocellular cancer: optimal strategies for screening and surveillance. Dig Dis 2009; 27: 142-147.

6 Llovet JM, Ricci S, Mazzaferro V, Hilgard P, Gane E, Blanc JF et al. Sorafenib in advanced hepatocellular carcinoma. N Engl J Med 2008; 359: 378-390.

7 Cheng AL, Kang YK, Chen Z, Tsao CJ, Qin S, Kim JS et al. Efficacy and safety of sorafenib in patients in the Asia-Pacific region with advanced hepatocellular carcinoma: a phase III randomised, double-blind, placebocontrolled trial. Lancet Oncol 2009; 10: 25-34.

8 Jungermann K, Kietzmann T. Zonation of parenchymal and nonparenchymal metabolism in liver. Annu Rev Nutr 1996; 16: 179-203.

9 Benhamouche S, Decaens T, Godard C, Chambrey R, Rickman DS, Moinard $\mathrm{C}$ et al. Apc tumor suppressor gene is the 'zonation-keeper' of mouse liver. Dev Cell 2006; 10: 759-770.

10 Hakvoort TB, He Y, Kulik W, Vermeulen JL, Duijst S, Ruijter JM et al. Pivotal role of glutamine synthetase in ammonia detoxification. Hepatology 2017; 65: 281-293.

11 Cadoret A, Ovejero C, Terris B, Souil E, Levy L, Lamers WH et al. New targets of beta-catenin signaling in the liver are involved in the glutamine metabolism. Oncogene 2002; 21: 8293-8301.

12 Mosmann T. Rapid colorimetric assay for cellular growth and survival: application to proliferation and cytotoxicity assays. J Immunol Methods 1983; 65: 55-63.

13 de La Coste A, Romagnolo B, Billuart P, Renard CA, Buendia MA, Soubrane $O$ et al. Somatic mutations of the beta-catenin gene are frequent in mouse and human hepatocellular carcinomas. Proc Natl Acad Sci USA 1998; 95: 8847-8851.

14 Park SY, Lee YK, Kim HJ, Park OJ, Kim YM. AMPK interacts with beta-catenin in the regulation of hepatocellular carcinoma cell proliferation and survival with selenium treatment. Oncol Rep 2016; 35: $1566-1572$.

15 Mao TL, Chu JS, Jeng YM, Lai PL, Hsu HC. Expression of mutant nuclear beta-catenin correlates with non-invasive hepatocellular carcinoma, absence of portal vein spread, and good prognosis. J Pathol 2001; 193: 95-101.

16 Dal Bello B, Rosa L, Campanini N, Tinelli C, Torello Viera F, D'Ambrosio $G$ et al. Glutamine synthetase immunostaining correlates with pathologic features of hepatocellular carcinoma and better survival after radiofrequency thermal ablation. Clin Cancer Res 2010; 16: 2157-2166.

17 Burke ZD, Reed KR, Phesse TJ, Sansom OJ, Clarke AR, Tosh D. Liver zonation occurs through a beta-catenin-dependent, c-Myc-independent mechanism. Gastroenterology 2009; 136: 2316-2324.e1-3.

18 van der Vos KE, Eliasson P, Proikas-Cezanne T, Vervoort SJ, van Boxtel R, Putker $\mathrm{M}$ et al. Modulation of glutamine metabolism by the PI(3)K-PKB-FOXO network regulates autophagy. Nat Cell Biol 2012; 14: 829-837.

19 Gebhardt R, Coffer PJ. Hepatic autophagy is differentially regulated in periportal and pericentral zones - a general mechanism relevant for other tissues? Cell Commun Signal 2013; 11: 21. 
20 Kabeya Y, Mizushima N, Ueno T, Yamamoto A, Kirisako T, Noda T et al. LC3, a mammalian homologue of yeast Apg8p, is localized in autophagosome membranes after processing. EMBO J 2000; 19: 5720-5728.

21 Shi YH, Ding ZB, Zhou J, Hui B, Shi GM, Ke AW et al. Targeting autophagy enhances sorafenib lethality for hepatocellular carcinoma via ER stressrelated apoptosis. Autophagy 2011; 7: 1159-1172.

22 Prieto-Dominguez N, Ordonez R, Fernandez A, Garcia-Palomo A, Muntane J, Gonzalez-Gallego J et al. Modulation of autophagy by sorafenib: effects on treatment response. Front Pharmacol 2016; 7: 151.

23 Lock R, Roy S, Kenific CM, Su JS, Salas E, Ronen SM et al. Autophagy facilitates glycolysis during Ras-mediated oncogenic transformation. Mol Biol Cell 2011; 22: 165-178.

24 Hart LS, Cunningham JT, Datta T, Dey S, Tameire F, Lehman SL et al. ER stress-mediated autophagy promotes Myc-dependent transformation and tumor growth. J Clin Invest 2012; 122: 4621-4634.

25 Elgendy M, Sheridan C, Brumatti G, Martin SJ. Oncogenic Ras-induced expression of Noxa and Beclin-1 promotes autophagic cell death and limits clonogenic survival. Mol Cell 2011; 42: 23-35.

26 Guo JY, Chen HY, Mathew R, Fan J, Strohecker AM, Karsli-Uzunbas G et al. Activated Ras requires autophagy to maintain oxidative metabolism and tumorigenesis. Genes Dev 2011; 25: 460-470.
27 White E. Deconvoluting the context-dependent role for autophagy in cancer. Nat Rev Cancer 2012; 12: 401-410.

28 Marino G, Niso-Santano M, Baehrecke EH, Kroemer G. Self-consumption: the interplay of autophagy and apoptosis. Nat Rev Mol Cell Biol 2014; 15: 81-94.

(c) (1) (2)(2) This work is licensed under a Creative Commons At 1 is $\mathrm{si}$ Ation-NonCommercial-ShareAlike 4.0

International License. The images or other third party material in this article are included in the article's Creative Commons license, unless indicated otherwise in the credit line; if the material is not included under the Creative Commons license, users will need to obtain permission from the license holder to reproduce the material. To view a copy of this license, visit http://creativecommons.org/licenses/by-nc-sa/4.0/

(C) The Author(s) 2018 